\title{
médecine/sciences : un trait d'union
}

Voici le premier numéro de médecine/sciences, mensuel de communication scientifique francophone de vocation internationale.

Cette nouvelle revue répond à une ambition principale : établir un dialogue entre chercheurs et cliniciens. Bien entendu, cette ambition ne trouvera son originalité qu'au prix d'une double attention : celle de la rigueur, pour satisfaire les plus exigeants des fondamentalistes, et celle de la clarté pour s'attacher les praticiens les plus surchargés.

médecine/sciences est un outil de transmission des connaissances pour les étudiants des deuxième et troisième cycles, les internes des hôpitaux, les enseignants de biologie, de médecine, de pharmacie, les ingénieurs des biotechnologies...

médecine/sciences publie des articles dans les domaines suivants :

la recherche dans toutes les disciplines biologiques et médicales (biologie cellulaire et moléculaire, investigations cliniques, épidémiologie);

les implications médicales potentielles des autres disciplines scientifiques (physique, chimic, pharmacie, anthropologie, éthique, psychologie, économie, sociologie...).

La singularité de médecine/sciences réside dans le critère de choix de son contenu : les articles de

Jean-François Lacronique Rédacteur en chef, France. synthèse, par exemple, sont à la fois compréhensibles par tous, mais aussi crédibles pour les spécialistes parce qu'ils sont écrits par des auteurs de haute qualité scientifique. Il en va de même pour les autres rubriques : hypothèses et débats, notes originales, éditoriaux et nouvelles d'actualité scientifique internationale.

Cette revue est née de la volonté de chercheurs et de médecins qui sont -régulièrement confrontés au problème de l'information scientifique de haut niveau, actuellement diffusée presqu'exclusivement en langue anglaise; ils souhaitent disposer, avec médecine/sciences, d'un équivalent en français des meilleures revues de synthèse angloaméricaines.

La revue a reçu l'encouragement déterminant des gouvernements français et québecois, matérialisant ainsi sa vocation internationale et culturelle.

Une science dont l'universalité s'établirait par l'intermédiaire d'une langue exclusive perdrait non seulement de son pouvoir, mais aussi de sa richesse. La diversité dans l'expression scientifique, loin de constituer un élément de dispersion désuet ou chauvin, doit être une source de diffusion de la connaissance. Mais là s'arrête l'esprit militant : la qualité scule sera le moteur de la diffusion de médecine/sciences. Sachez la reconnaître, l'encourager, y contribuer.

Michel Bergeron

Rédacteur en chef, Québec. 